\title{
Using the flexibility of EPS as a means of improving the system reliability of power systems in modern conditions
}

\author{
Alexey Osak ${ }^{1, *}$, Elena Buzina $^{1}$ \\ ${ }^{1}$ ESI SB RAS, 664033 Lermontov str., 130, Irkutsk, Russia
}

\begin{abstract}
Scientific research in the field of EPS flexibility has recently been intensified, and this flexibility is considered in a fairly broad setting. It is important to note that the issues of EPS flexibility are not new, but the term "flexibility" was not used to refer to this issue before, although all the main issues were considered within the framework of the topic of system reliability and development of EES, and the issue of continuity of modern research with previous scientific research and existing scientific results is important. Traditionally, one of the aspects of system reliability has been the power security of the EPS, which refers to the ability of the system to withstand sudden disturbances without unintended impacts on electricity consumers. At the same time, the analysis of power security was mainly performed only in the volume of the system-forming power gird of the EPS. For the power systems of the future, the importance of power security increases significantly, but as an analysis of the power security of the system-forming and distribution power grids together. For a number of years, the authors have been engaged in research in the field of mode reliability using the method of calculating EPS modes taking into account discrete and interval characteristics of mode parameters. This method allows us to study the properties of controllability of power systems of the future in the required problem statement.
\end{abstract}

\section{Introduction and problem statement}

Recently, scientific research in the field of Electric Power System (EPS) flexibility has been intensified, and this flexibility is considered in a fairly broad setting [1, 2]. In accordance with [3]: Flexibility describes the degree to which a power system can adjust the electricity demand or generation in reaction to both anticipated and unanticipated variability. Flexibility indicates the capacity of a power system network to reliably sustain supply during transient and large imbalances. A technoeconomic definition by International Energy Association states that, power system flexibility is the ability of a power system to reliably and cost-effectively manage the variability and uncertainty of demand and supply across all relevant timescales, from ensuring instantaneous stability of the power system to supporting long-term security of supply $[4,5]$.

It is important to note that the issues of EPS flexibility are not new. Previously, the term "flexibility" was not used to refer to this issue, although all the main issues were considered within the framework of the topic of systems reliability and development of EPS [6-8]. Accordingly, the issue of continuity of modern research with previous scientific research and existing scientific results is important.

In the traditional electric power industry, the main emphasis in the field of system reliability of power plants was placed on the maneuverability of highcapacity generating equipment and sufficient reserves of capacity of inter-system and intra-system main power grids. The nature of load changes over time (over a year, over a week, over a day, over an hour, over a minute) was fairly predictable and was determined by the types of consumers, including the type of production at an industrial enterprise. Unscheduled deviations from the forecast mode were also predictable, because the main reason for them was an emergency shutdown of equipment, which allowed us to normalize such perturbations (normative perturbations in stability analysis [9]), and also allowed us to apply the rich experience of studying the hardware reliability of elements of the EPS [10]. In such conditions, it was possible to plan and implement the required properties of the power plant quite effectively, even in the long term, both in terms of the controllability of power grid elements and the maneuverability of generating objects. To ensure system reliability, the tasks of determining the optimal volumes of hot and cold reserves at power plants were solved. These aspects, in fact, provided the required flexibility of the EPS (primarily due to largescale generation and main electric grids), and the effectiveness of the traditional approach within the previous technological structure has been confirmed by practice.

In modern conditions, when there is a mass construction of small power plants using renewable energy sources, the volume of installation of power storage devices is increasing, consumer electrical installations are changing (they are becoming adaptive

Corresponding author: osakalexey@mail.ru 
and intelligent), there is a process of mass appearance of electric vehicles, and it is necessary to review traditional provisions in the field of system reliability. The problem is caused by the sharply variable and unreliable predictable of nature of the EPS loads curve, since the EPS load capacity is defined as the difference between the current load of electric receivers and generators power of consumers. Small utility consumers are not involved in operational dispatch control and the prospect of their involvement in the future is unlikely. As a result, there is no dialogue between consumers and energy companies, so the reasons and motives for changing consumer loads, including abrupt ones, are unknown to energy companies. In the case of an active consumer with its own generation using renewable energy sources, there may be a situation of a sharp transition from the mode of issuing electricity to the grid to the mode of maximum load consumption and oppositely, and this will happen without notifying energy companies.

Special attention should be paid to the development of electric transport (electric vehicles). If the entire current fleet of the country is converted from internal combustion engines to electric drive, it will require about a 3-fold increase in power generation. Of course, the scenario of $100 \%$ switching to electric transport is unlikely, but it is also quite likely that the transfer of $10 \%$ of vehicles to electric drive will require a $20 \%$ increase in electricity consumption, and at the expense of increasing the load on the distribution grids.

Therefore, the traditional approach, where the flexibility of EPS was provided at the level of reserves of large-scale power plants and main electric grids with the general passivity of distribution grids, will not ensure the system reliability of the power systems of the future. It is not a problem to provide the required reserve in the future at the level of large-scale power plants and capacity of main electric grids, the problem will be in the transmission and distribution of electricity to consumers. It will require either a multiple increase in the capacity of distribution grids, or the use of new approaches to ensure system reliability due to the flexibility of the EPS on the part of distribution grids and active consumers.

In addition to new trends with active consumers, electric transport and distributed low-power generation, there are also traditional problems of covering peak loads, as well as the problem of maintaining the parameters of the electric regimes within acceptable limits in repair and post-emergency regimes in EPS. In the cold season, the maximum load is associated with electric heating (as the main or additional means of heating), in the warm season, the maximum load is associated with cooling (air conditioning). In Russia, there are regions, in particular the Irkutsk region, where the share of electric heating is very high, including many localities where electric heating is the dominant method of heating premises. Analysis of the load schedules of real power systems shows that during periods of cooling, which in total amount to about 1-3 weeks per year, there is almost a 2-3-fold increase in loads in distribution grids, compared with the average level of loads in the autumn-winter period.
Accordingly, the transmission capacity of distribution electric grids is selected based on the maximum consumption (maximum load) and nonexceeding of the long-term permissible current of the grids elements in the event of a possible failure of any one grids element (criterion "N-1"). Due to the lack of automation tools, distribution grids are traditionally built as a radial grid with branches and a small number of cross-links forming rings. For this reason, for mediumvoltage distribution grids, reliability requirements against the background of the rare use of ring circuits, leads to the fact that the power supply to consumers is usually carried out by two parallel power lines (air or cable). As a result, in a normal scheme, even in the maximum load mode, grids elements (power lines and transformers) are loaded no more than $50 \%$, and at the average annual load level, the load of grids elements usually does not exceed $15-30 \%$ of the permissible value. In cities, medium-voltage power lines are mostly made with expensive cable lines with a long repair period if it is damaged. In rural areas with low population density, medium-and low-voltage power lines are long. Both factors significantly increase the unit cost of urban and rural power lines per unit of electricity consumed. Thus, the capacity of the distribution grid is excessive, which increases the capital and operating costs for the construction, reconstruction and operation of electric grid, which are included in the electricity transmission tariff.

If the load increases, which may be caused by the construction of new residential or non-residential facilities, or the mass appearance of new electric receivers of significant total capacity, such as electric vehicles (if their total number increases), the existing radial structure of the electric grid will not allow using the existing excess capacity of the grid. Therefore, it is necessary to build new or increase the capacity of existing electric transformer substations and power lines, which in turn will lead to an increase in electricity transmission rates. While maintaining the traditional approach, the new transformers and power lines will also operate in low-load mode most of the time. Thus, the effectiveness of the traditional approach is quite low, which is paid for by consumers.

Since its inception, distribution electric grids are nonautomated and poorly observed objects, in contrast to the main electric grids and power supply grids of the most responsible industrial enterprises. This fact is due to the high cost, low reliability and low efficiency of the automation and remote control devices available at that time. Over the past decades, there has been a rapid and high-quality leap in the development of computer technology and digital communications, which allows you to create affordable, highly efficient automation tools at any level. Accordingly, there is a prerequisite for mass automation, intellectualization and digitalization of distribution grids, since this will reduce the cost of developing distribution grids.

For the electricity industry of the future it is crucial to select the optimal regimes control in normal conditions, and the control of emergency and forced regimes. Under normal conditions, the main way to 
influence active consumers is economic motivation, when due to discounts and surcharges to the price of electricity, a large number of active consumers are encouraged to manage their consumption according to the optimal scenario. At the same time, unlike traditional wholesale and retail markets (B2B market), the main players are quite small active consumers (B2C market), who will be ready only for simple rules of the game with a minimum amount of formal requirements and reporting documentation for them. It is important to understand that these objective circumstances will impair the reliability of the forecast for the control of EPS regimes.

The situation is even more complicated for active consumers with emergency and forced regimes in the EPS. The main players are small consumers, who will not be involved in operational dispatch control. accordingly, energy companies will not be able to get information about the technical condition, accidents and repairs of such active consumers. If the high-power generating equipment, as well as the equipment of power grid companies, have requirements for reliability, requirements for their operating conditions in emergency modes, as well as requirements for their readiness in maximum load modes (readiness to pass the autumnwinter period), then the low-power generation equipment, as well as electrical installations for managing the mode of active consumers, can not be normalized in terms of the requirements for their operation in emergency modes, as well as the requirements for their readiness in maximum load modes. Accordingly, there is a very high probability of mass failure of small generation and controlled elements of active consumers during emergency and post-accident modes in the EPS (electromechanical and transient processes, voltage and frequency deviations), as well as under extreme climatic factors. Accordingly, it is impossible to talk about the guarantee of the volume of system services provided by active consumers. It can be assumed that under normal conditions, most of the time active consumers will manage their consumption according to a relatively optimal scenario in accordance with the factors of economic incentives from energy companies. But there will also be time periods when active consumers will lose their activity, or will not act according to the optimal scenario accordingly, the EPS should be prepared for such a turn of events, ensuring both system reliability and reliability of power supply to consumers. Therefore, the key feature of the future power systems is the ability to operate in wide operating conditions, including forced operation of individual elements and sections of the distribution grid.

It is assumed that the greatest economic effect of digitalization and intellectualization of electric distribution grids will be in the following aspects:

- in the wide use of ring modes of distribution grids, with automatic or automated control of the ring grid topology,

- automatic control of the reactive power regimes in a wide range (maintaining optimal voltage levels in any circuit-mode situations),
- in the managing the demand of active consumers, including through electric vehicles (coordinated management of the charging process of electric vehicles and their use as energy storage devices in emergency situations).

\section{Power system security in new conditions}

Traditionally, one of the aspects of system reliability of EPS has been the power system security, which refers to the ability of the system to withstand sudden disturbances without unintended impacts on electricity consumers. At the same time, the analysis of power system security was mainly performed only in the volume of the system-forming grid of the EPS. For the power systems of the future, the importance of power system security increases significantly, but as an analysis of the power system security of a jointly system-forming and distribution grids.

The authors propose to use an iterative two-level system for calculating and optimizing electrical regimes, where information is exchanged at the intersection of levels not by specific values of parameters of electrical modes, but by acceptable ranges and optimal (target) values of parameters of electrical regimes. This approach methodically brings together the traditional approach, where the system reliability of the EPS was ensured by optimal volumes of hot and cold reserves at power plants, with a new approach to managing the flexibility of the EPS. For the energy of the future, the managed resource is not only the range of possible generation capacity at large power plants (taking into account the hot and cold reserve), but also the range of controlled changes in load capacity in distribution grids.

The authors have been engaged in research in the field of power system security for a number of years. To solve this problem, it is proposed to use the method of calculating EPS regimes repeatedly tested on other tasks, taking into account the discrete and interval characteristics of the regimes parameters $[11,12]$. This method allows us to study the controllability properties of future power systems in the required task statement.

In general, the balanced steady state parameters as well as their functions should be as close as possible to values $V \rightarrow \hat{V}$, these values are defined as discrete characteristics of the regimes parameters. They can be measurements, planned values of regimes parameters, forecast values, etc. Elements of $v_{i} \in V$ are characterized by two parameters $\left\{\hat{v}_{i}, \hat{\sigma}_{i}\right\}$. In the case of measurement, $\hat{\sigma}_{i}$ means the dispersion of $\hat{v}_{i}$, in other cases, it may be the permissible deviations of $v_{i}$ from $\hat{v}_{i}$, specified as a percentage.

In addition, the $G$ regimes parameters should be in some intervals of their $G \rightarrow[\underline{G}, \bar{G}]$ values, if possible. Accordingly, each interval variable $g_{i}$ is 
characterized by four parameters $\left\{\underline{g}_{j}, \underline{\sigma}_{j}, \bar{g}_{j}, \bar{\sigma}_{j}\right\}$, where $\underline{g}_{j}, \bar{g}_{j}-$ upper and lower interval limits; $\underline{\sigma}_{j}$, $\bar{\sigma}_{j}-$ boundary dispersions. From $\underline{\sigma}_{j}, \bar{\sigma}_{j}=0$ it follows that $\underline{g}_{j} \leq g_{j} \leq \bar{g}_{j}$. The control range of controlled items (see Item 2), security restrictions (see Item 3-4), the volume control actions (see Item 5-6) are specified using these interval limits.

The current load and generation values, as well as various expected (recommended, optimal) values of voltages, generations and loads of nodes, currents and flows through the branches can be set either by discrete parameters of the $V$, or by interval parameters of $G$ with the values of dispersions (relative deviations) selected by taking into account the ranking of the importance of the recommended constraints or the severity of the control actions. By setting the current load and the active power of the generation by the discrete parameters of $V$, in the simulation of the emergency perturbation first of all will be found the controlling effects, not related to the change in the load and the generation of active power. And only if it is impossible to bring the regimes into the permissible area, the resource controlling effects will be used to reduce the load and change the generation.

Because of the need to rank the role of variables $y_{i}$ in the functioning of the system, it is necessary to take them into account repeatedly in the $G$ with different boundary characteristics and percentage rates $\left(\underline{\sigma}_{i j}, \bar{\sigma}_{i j}\right)$. Accordingly, the wider the range, the lower the percentage rates. The possibility of simultaneous assignments to the same parameter regime of restrictions in the form of discrete values of the expected values of $V$ and several interval ranges $G$ with different values of variance (percentage variance) makes it quite flexible to influence the results of the calculation of the variation of the controlling effects. Using this method, it is possible to perform the analysis of PS security, assessing the control of PS and finding different ways of automatic or operational control allowing PS to move from preemergency to acceptable post-emergency mode in the event of emergency disturbances.

To characterize the proximity measure of $V \rightarrow \hat{V}$, $G \rightarrow[\underline{G}, \bar{G}]$, the weighted least squares function is commonly used:

$$
f=\sum_{i}^{n} a_{i}^{2}+\sum_{i}^{m} b_{i}^{2}
$$

where $a_{i}=\frac{v_{i}-\hat{v}_{i}}{\hat{\sigma}_{i}} \cdot k_{v i}$;

$$
b_{i}=\frac{g_{i}-g_{i}^{*}}{\sigma_{i}^{*}} \cdot k_{g i} \cdot k_{i} \text {; }
$$

The coefficients $k_{v i}, k_{g i}$ take into account the regulating effect of interval variables on the variables $v_{i}$ и $\mathrm{g}_{\mathrm{i}}$. If there is a regulating effect of some interval variable $\mathrm{g}_{\mathrm{j}}$ on another variable, for example, $v_{i}$, it exists if the variable $g_{j}$ does not reach one of the boundaries. Therefore $k_{v i_{g i}}=1-k_{j}$.

In the accepted approach, $0 \leq d\left(g_{i}\right) \leq 500$ depends on the distance of $g_{i}$ from the interval boundaries, on the value of $f$ and on the course of the computational process.

$$
\begin{gathered}
g_{i} \leq \underline{g}_{i} \rightarrow\left\{k_{i}=1 ; g_{i}^{*}=\underline{g}_{i} ; \sigma_{i}^{*}=\underline{\sigma}_{i}\right\} ; \\
g_{i} \geq \bar{g}_{i} \rightarrow\left\{k_{i}=1 ; g_{i}^{*}=\bar{g}_{i} ; \sigma_{i}^{*}=\bar{\sigma}_{i}\right\} ; \\
\underline{g}_{i}<g_{i}<\bar{g}_{i} \rightarrow\left\{\begin{array}{l}
g_{i}^{*}=1 ; g_{i}^{*}=g_{i} ; \sigma_{i}^{*}=\underline{\sigma}_{i} \cdot \alpha+\bar{\sigma} \cdot(1-\alpha) ; \\
0<\alpha<1 ; \\
k_{i}=\frac{1}{1+d\left(g_{i}\right)}
\end{array}\right\} ;
\end{gathered}
$$

The steady state of PS is usually described by the equations of balance of currents or powers in the grid nodes and in vector form:

$$
W(X, Y)=0
$$

here, the vector $Y$ is a vector of independent parameters of the regime, which at the stage of solving the formula (2) take specific values. $X$ is the vector of dependent parameters on the vector $Y$. This implicit dependency is indicated by:

$$
X=X(Y) \text {. }
$$

The solution of the problem is reduced to finding such a vector $Y$, at which:

$$
\begin{gathered}
\min f(Y) \\
\text { and } \\
w(X, Y)=0 .
\end{gathered}
$$

Sequential calculations are performed using the above method both for the system-forming grid and for each section of the distribution grid (connected to the main grid, but not connected to other sections of the distribution grid) with a search for regulatory disturbances. On the basis of each calculation, the required intervals of boundary regimes parameters at the junction of the system-forming and distribution network are determined. If you cannot enter regimes parameters in a valid region, requires the modification of the adjustment ranges of controlled parameters, which allows to determine optimal automatic control options, and if that's not enough, the definition of requirements for the development of the means of control of this part of the grid.

\section{Conclusion}

In this study, the authors made the following conclusions: 
1. The task of ensuring EPS flexibility is a development of the traditional tasks of ensuring system reliability. Therefore, it is necessary to ensure the continuity of modern research in the field of EPS flexibility with previous scientific research and existing scientific results.

2. Researchers in the field of EPS flexibility should develop their research areas in order to harmonize the newly introduced terminology and concepts with existing developments in the field of system reliability.

3. Researchers in the field of system reliability should develop their research areas to take into account current trends in distribution grids, accounting for small power generation and active consumers.

4. The Flexibility of the power systems of the future, in its balance aspect, will be provided largely due to active consumers and distributed generation of low power, including installed at consumers. But, these processes have not led to lower system reliability of the EPS or reduction of reliability of power supply of consumers, requires the development of distribution grids in terms of their observability, to provide automatic flow control of active and reactive power (banding grids, provision of reactive power sources, provision and installation of a automatic of regimes and emergency control).

5. For the power systems of the future, the importance of power system security increases significantly, but as an analysis of the power system security of a jointly system-forming and distribution grid. It is proposed to use an iterative two-level system for calculating and optimizing electrical regimes, where information exchange between levels is carried out at the interface in the form of transmitting acceptable ranges and optimal (target) values of regimes parameters. Mathematical and software tools for solving the problem in this setting are proposed.

\section{ACKNOWLEDGEMENTS}

The work was carried out within the project III.17.4.2 (No. AAAA-A17-117030310438-1) of the fundamental research program of the Siberian Branch of the Russian Academy of Sciences and partly supported by the Russian Science Foundation, Grant No. 19-49-04108.

\section{References}

1. Cochran, J., Miller, M., Zinaman, O., Milligan, M., Arent, D., Palmintier, B., O'Malley, M., Mueller, S., Lannoye, E., Tuohy, A., Kujala, B., Sommer, M., Holttinen, H., Kiviluoma, J., and Soonee, S. K. "Flexibility in 21st Century Power Systems", United States, 2014, doi:10.2172/1130630.

2. N. Voropai et al., "The Development of a Joint Modelling Framework for Operational Flexibility in Power Systems", 16th Conference on Electrical Machines, Drives and Power Systems (ELMA), Varna, Bulgaria, 2019, pp. 1-6, doi: 10.1109/ELMA.2019.8771685.
3. Babatunde O. M., Munda J. L., Hamam, Y. (2020). "Power system flexibility", A review. In Energy Reports, Elsevier Ltd, 2020, Vol. 6, p. 101-106, doi:10.1016/j.egyr.2019.11.048.

4. "Status of Power System Transformation 2018: Advanced Power Plant Flexibility", IEA, Paris, 2018, doi:10.1787/9789264302006-en.

5. "Status of Power System Transformation 2019: Power system flexibility", OECD Publishing, Paris, 2019, doi: 10.1787/7c49400a-en.

6. Ed. N.A. Manov, "Methods and models for studying power system reliability", Syktyvkar: Komi SC UrB RAS, 2010, 292 p. (in Russian).

7. Eds. N.I. Voropai, G.F. Kovalev, "Concept of reliability control in electric power engineering", M.: LLC PH "Energy". 2013. 304 p. (in Russian).

8. Eds. N.I. Voropai " Reliability of energy systems: problems, models and methods of their solution", Novosibirsk, Nauka, 2014, 284 p. (in Russian).

9. "Requirements for ensuring the reliability of electric power systems, reliability and safety of electric power facilities and power receiving installations "Guidelines for the stability of power systems", approved by the Order of the Ministry of energy of the Russian Federation No. 630 dated 03.08.2018. (in Russian).

10. Nepomnyashchiy V.A., Daryan L.A. "Reliability of equipment of electric networks 220-750 kV power systems", Moscow, Energoprogress: Energetik, 2018, 123 p. (in Russian).

11. Osak A., Panasetsky D., Buzina E. "Methods of analysis of the power system security", E3S Web Conf, Volume 58, 2018, Article Number 02018, doi: 10.1051/e3sconf/20185802018.

12. Osak A., Panasetsky D., Buzina E. "Method of analysis of power system security for studying the properties of adaptability in normal and emergency regimes", Problems of energy and sources saving. Tashkent, №3-4. pp. 60-68. (in Russian). 